\title{
Recent advances in the management of osteoporosis
}

\author{
Juliet Compston
}

\section{Introduction}

Osteoporotic fractures are a major cause of morbidity and mortality in the elderly population. One woman in two and one man in five over the age of 50 years will sustain a fracture during their remaining lifetime, resulting in an annual cost to the health services in the UK of around $\mathfrak{E} 2$ billion. In recent years there have been major advances in the prediction of fracture risk, enabling more accurate targeting of treatment. Furthermore, a range of therapeutic options with proven anti-fracture efficacy is now available. In spite of these advances, however, management of osteoporosis remains suboptimal and only around one-third of patients presenting with a fragility fracture receive appropriate diagnostic assessment and therapeutic intervention.

\section{Diagnosis of osteoporosis and assessment of fracture risk}

In 1994 a World Health Organization (WHO) study group produced an operational classification of osteoporosis, based on bone mineral density (BMD) measurements as assessed by dual energy X-ray absorptiometry (DXA). ${ }^{1}$ Osteoporosis was defined as a BMD T-score of $\leq-2.5$, the T-score being the number of standard deviations above or below the mean value for normal young women. Osteopenia was defined as a T-score between -1 and -2.5 , while severe or established osteoporosis was defined as a T-score $\leq-2.5$ in the presence of one or more fragility fractures. These definitions were based on the known inverse relationship between BMD and fracture risk.

The use of BMD measurements to predict future fracture risk has a high specificity but a low sensitivity, and the majority of postmenopausal women presenting with a fragility fracture have a BMD T-score higher than -2.5 . Recently the importance of clinical risk factors that affect fracture risk independently of BMD has been recognised. ${ }^{2}$ These are shown in Table 1 and are used in the WHO-supported FRAX $^{\circledR}$ risk algorithm, with or without femoral neck BMD. ${ }^{3}$ FRAX ${ }^{\circledR}$ expresses fracture risk as the 10-year probability of hip fracture and of major osteoporotic fracture (hip, wrist, spine or humerus), from which intervention thresholds can be derived. Country-specific versions of FRAX $^{\circledR}$ are available for a number of countries, including the UK (Fig 1; www.shef.ac.uk/FRAX).

Juliet Compston, professor of bone medicine, University of Cambridge School of Clinical Medicine, Cambridge

This talk was part of the conference 'Expounding boundaries of endocrinology' which took place at the Royal College of Physicians on 27 January 2009 and was organised by Professor John Wass, Oxford Centre for Diabetes, Endocrinology and Metabolism
It should be noted that $\operatorname{FRAX}^{\circledR}$ has some limitations. It is designed only for postmenopausal women and men over the age of 40 who have not previously received bone protective therapy. It uses only 'yes' or 'no' responses, and so does not take account of dose-responses for several risk factors including previous fracture, glucocorticoid therapy and smoking. The weighting given to any previous fragility fracture is the same and prior clinical vertebral fractures, which carry a higher risk than other previous fractures, are not considered separately. Falls are not included in the algorithm. For all these reasons, it is important to exercise clinical judgement when using FRAX $^{\circledR}$ to assess fracture risk in clinical practice.

\section{Case finding strategy}

Population-based screening of postmenopausal women and older men cannot be justified on economic grounds and current guidelines recommend an opportunistic case finding strategy, in which postmenopausal women and older men with one or more clinical risk factor undergo fracture risk assessment using FRAX ${ }^{\circledR}$. In individuals with very low or very high fracture probability, decisions about treatment can often be made on the basis of clinical risk factors without recourse to BMD but in those at intermediate risk, BMD is helpful in improving the estimation of fracture probability.

\section{Intervention thresholds - who should be treated?}

Intervention thresholds should be determined by clinical considerations, but should also be cost effective. The National

Table 1. Risk factors for osteoporosis.

\begin{tabular}{ll} 
BMD-independent & Other \\
\hline Age & Hypogonadism \\
Previous fracture & Endocrine disease \\
Glucocorticoids & Gastrointestinal disease \\
Family history & Chronic renal disease \\
Low BMI & Immobiiity \\
Smoking & Organ transplantation \\
Rheumatoid arthritis & Drug-induced \\
Alcohol abuse & Aromatase inhibitors \\
Falls & Androgen deprivation Rx PPls \\
& Thiazolidenediones \\
& SSRls
\end{tabular}

$\mathrm{BMD}=$ bone mineral density; $\mathrm{BMI}=$ body mass index; $\mathrm{PPI}=$ proton pump inhibitors; SSRls = selective serotonin re-uptake inhibitors. 
Fig 1. The World Health Organization fracture risk assessment tool (FRAX $\left.{ }^{\circledR}\right)$. Data have been entered for a 55-year-old woman with a previous fracture. The 10-year probability of sustaining a major osteoporotic fracture is $9.6 \%$ and of a hip fracture $1.5 \%$. Image used with permission of the WHO Collaborating Centre for Metabolic Bone Diseases, University of Sheffield. FRAX ${ }^{\circledR}$ is registered to Professor JA Kanis, University of Sheffield.
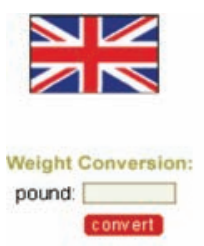

Height Conversion: inch

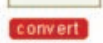

Osteoporosis Guideline (NOG) uses thresholds that correspond to the risk of fracture in a woman with a prior fracture, irrespective of BMD. ${ }^{4}$ These intervention thresholds are cost effective and are consistent with the guidance previously produced by the Royal College of Physicians (RCP). ${ }^{5,6}$ The percentage of the population potentially eligible for treatment rises from $23 \%$ in 50-54 year old women to $46 \%$ in women aged 80-84 years.

When the UK version of FRAX ${ }^{\circledR}$ is used, the estimated 10 -year fracture probability can be electronically transferred, using the NOG guidance button, to charts containing the intervention thresholds (Fig 2; www.shef.ac.uk/FRAX/NOGG). It should be emphasised that these thresholds serve as a reference upon which to make treatment decisions, but do not replace clinical judgement when dealing with individual patients.

\section{Pharmacological intervention}

A number of treatments have been shown to reduce fracture risk in postmenopausal women. ${ }^{7}$ These include the bisphosphonates,

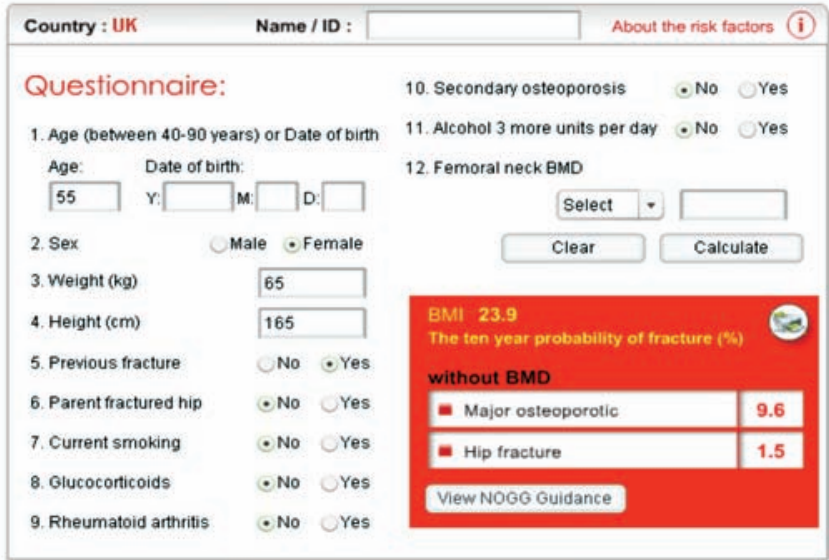

raloxifene, strontium ranelate and parathyroid hormone peptides. All have been shown to reduce vertebral fractures but evidence for reduction in non-vertebral and hip fractures is not universal (Table 2). ${ }^{7}$ The reduction in vertebral fracture rate has generally been between $50-70 \%$ and since there have been no head-to-head studies with fracture as the primary outcome, direct comparison of efficacy between agents is not possible. The magnitude of reduction in non-vertebral fracture, where demonstrated, has generally been smaller and in the order of $15-25 \%$. This is likely to reflect, at least in part, the importance of falls in the pathogenesis of these fractures but may also result from differences in the effects of the various treatments on cortical versus cancellous bone.

Because of their broad spectrum of anti-fracture efficacy alendronate, risedronate, zoledronate and strontium ranelate are generally regarded as front-line treatments in the majority of postmenopausal women. However, since generic versions of alendronate are now available, they represent the most cost-effective intervention and are therefore used as the first option in most cases. ${ }^{8}$
Fig 2. Assessment and treatment thresholds in the absence of a bone mineral density (BMD) test (left) and with a BMD test to compute fracture probability (right) for men and women. The graph on the left is used when BMD measurements are not included in the FRAX ${ }^{\circledR}$ assessment; the orange area represents a range of intermediate risk in which BMD measurement is recommended, while for fracture probabilities in the green or red area, no further assessment is required. When BMD has been included in the FRAX ${ }^{\circledR}$ assessment, the graph on the right-hand side is used to determine whether fracture probability is above or below the intervention threshold. Reproduced with kind permission from Springer Science + Business Media. ${ }^{4}$
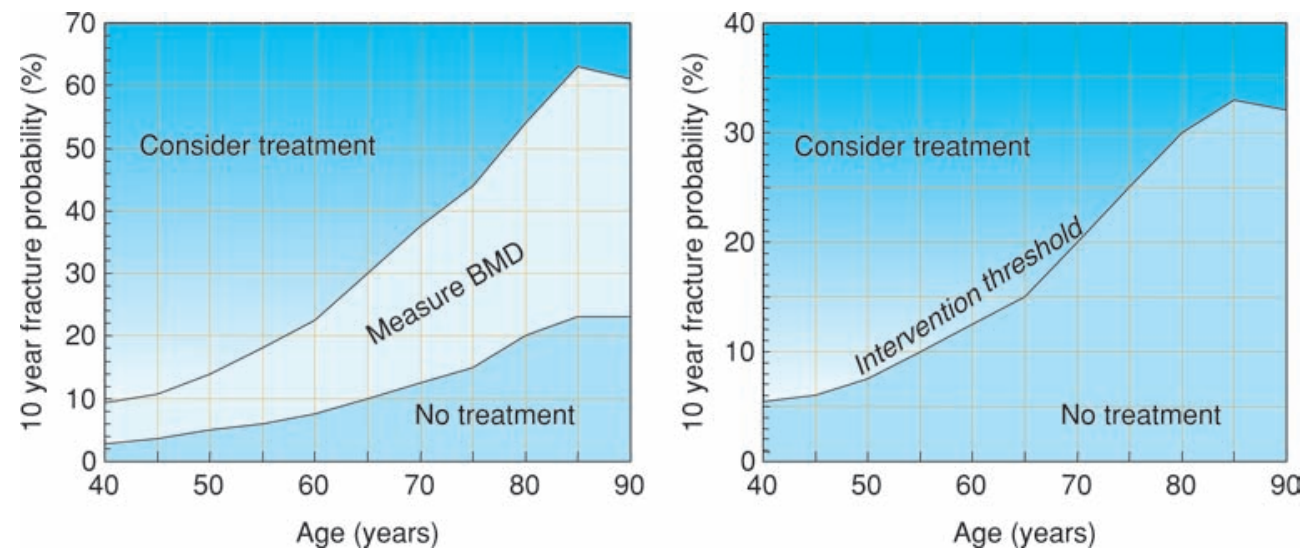


\section{Bisphosphonates}

Bisphosphonates are analogues of inorganic pyrophosphate and inhibit bone resorption. Nitrogen-containing bisphosphonates, which include alendronate, risedronate, ibandronate and zoledronate, act on the mevalonate pathway by inhibiting the action of farnesyl diphosphate and thus preventing the prenylation of proteins essential for normal osteoclast function. Alendronate and risedronate are available as once daily or once weekly oral formulations, ibandronate as once monthly oral or once every three months intravenous (iv) formulations and zoledronate as a once yearly iv formulation. All of these bisphosphonates are approved for treatment of postmenopausal osteoporosis; alendronate, risedronate and zoledronate are also approved for men with osteoporosis and for glucocorticoidinduced osteoporosis.

Oral bisphosphonates have to be taken after an overnight fast and 30-60 minutes before the first food or drink of the day or any other medicinal products. The tablet must be swallowed with a glass of plain water and the patient should remain in the standing or sitting position for 30-60 minutes. Compliance with this dosing regimen is essential to maximise intestinal absorption and prevent the occurrence of upper gastrointestinal side effects. Oral bisphosphonates are therefore not suitable for very frail patients or those with cognitive dysfunction and are contraindicated in the presence of significant oesophageal disease. The most common side effect of iv bisphosphonates is an acute phase reaction following the injection or infusion. ${ }^{9}$ This is sometimes severe, but is self-limiting and can be avoided or reduced in severity by taking paracetamol on the day of the infusion and the subsequent one to two days.

The optimal duration of treatment with bisphosphonates is unclear. Withdrawal of alendronate after treatment periods of up to seven years is followed by resumption of bone loss in the hip although the time course of this may vary between bisphosphonates. ${ }^{10}$ However, there are potential concerns that long-

Table 2. Spectrum of anti-fracture efficacy of approved pharmacological interventions for osteoporosis.

\begin{tabular}{lccc} 
Intervention & Vertebral & Non-vertebral & Hip \\
\hline Alendronate & + & + & + \\
Risedronate & + & + & + \\
Zoledronic acid & + & + & + \\
Etidronate & + & - & - \\
Ibandronate & + & $+*$ & - \\
Raloxifene & + & - & - \\
PTH (1-84) & + & - & - \\
Teriparatide & + & + & $+*$ \\
Strontium ranelate & + & + & \\
& & & \\
${ }^{*}$ post-hoc analysis in high-risk group. \\
PTH = parathyroid hormone.
\end{tabular}

term suppression of bone turnover associated with treatment may eventually lead to adverse effects on bone strength. This remains largely a theoretical concern although there have been recent reports of atypical stress fractures in the femoral shaft or subtrochanteric region in patients on alendronate therapy; in some of these cases bone biopsies have been done and have shown severely suppressed bone turnover. ${ }^{11}$ However, it should be stressed that these fractures are extremely rare and easily outweighed overall by the beneficial effects of alendronate on fracture risk.

Another potential side effect of bisphosphonate therapy that has received much attention is osteonecrosis of the jaw. While it is likely that there is a causal association in patients with malignant disease receiving high doses of iv bisphosphonates, this remains unproven in patients receiving the much lower doses of bisphosphonates used for the treatment of osteoporosis. Since many of the cases reported have been associated with dental disease, invasive dental treatment should be completed before bisphosphonate therapy is started and where possible, avoided during treatment. ${ }^{12}$

\section{Strontium ranelate}

Strontium ranelate consists of two atoms of strontium linked to an organic acid, ranelic acid. Its mechanism of action is not fully understood but there is growing evidence that unlike other agents, its main effects are mediated through changes in bone material properties rather than alterations in bone remodelling. It is known that strontium can substitute for some of the calcium ions in hydroxyapatite and this may explain its effects on bone strength, rather than the weak effects that are seen on bone resorption and bone formation. ${ }^{13}$

Strontium ranelate is taken once daily as granules dissolved in water. It should be taken at least two hours after the last meal and is usually taken at bedtime. It does not cause upper gastrointestinal side effects and is therefore an alternative option in women who cannot tolerate oral bisphosphonates. It has a particularly strong evidence base in the very elderly, since unlike in most other clinical trials a large number of women over the age of 80 years were included in the phase III studies. Side effects include diarrhoea, nausea and headache; a small increase in the risk of venous thromboembolism was also seen in clinical trials.

\section{Raloxifene}

Raloxifene is a selective oestrogen receptor modulator and an inhibitor of bone resorption. It has been shown to reduce vertebral, but not non-vertebral, fractures and is taken orally as a single daily dose of $60 \mathrm{mg}$. Its use is associated with an increased risk of venous thromboembolism and it may cause or exacerbate vasomotor symptoms associated with the menopause. A small increase in the risk of stroke has also been reported. Its use is associated with a significant decrease in the risk of breast cancer. 


\section{Parathyroid hormone peptides}

When administered intermittently, parathyroid hormone (PTH) peptides have anabolic skeletal effects. The resulting increase in bone formation is most marked in cancellous bone; in cortical bone, the effects may be site specific and transient decreases in BMD are often seen in the first year or so of treatment, possibly due to increased cortical porosity. ${ }^{14} \mathrm{PTH}$ peptides are administered by once daily subcutaneous injection, in a dose or $20 \mu \mathrm{g}$ daily for teriparatide and $100 \mu$ g daily for PTH (1-84) and the duration of treatment is limited to 18 and 24 months respectively. Because of their expense, the use of these drugs is restricted to individuals with severe osteoporosis in whom other treatments are not tolerated or appear to be ineffective.

\section{Calcium and vitamin $D$}

Calcium and vitamin D supplements should be prescribed as an adjunct to other treatments unless there is evidence of an adequate dietary calcium intake and normal vitamin D status. The recommended daily doses are 1-1.2 $\mathrm{g}$ and $800 \mathrm{IU}$ respectively. Because of their high risk of vitamin D deficiency, supplementation at these levels is recommended in the elderly housebound population and those living in residential care homes or nursing homes.

\section{Current guidance for the management of osteoporosis}

The National Institute for Health and Clinical Excellence (NICE) has produced guidance for the primary and secondary prevention of osteoporotic fractures. ${ }^{15,16}$ This guidance is restricted to postmenopausal women with osteoporosis as defined by a BMD T-score of $\leq-2.5$, and does not include men with osteoporosis or individuals treated with glucocorticoids.
Several recently approved interventions, including ibandronate and zoledronate, are also not included in the guidance. The NOG was launched in October 2008 to address these deficits. ${ }^{17}$ It is endorsed by many scientific and professional organisations including the National Osteoporosis Society and the RCP.

Both NICE and NOG guidance include recommendations to treat elderly postmenopausal women with a fragility fracture and to use generic alendronate as a first-line option because of its high cost effectiveness. There is also agreement that BMD measurements may be useful in reaching treatment decisions in younger postmenopausal women with a fragility fracture. However, whereas NICE requires a T-score $\leq-2.5$ in most women for either primary or secondary prevention, NOG recognises the added contribution of independent clinical risk factors to fracture prediction and recommends the use of the WHO-supported fracture risk algorithm FRAX ${ }^{\circledR}$. In addition, NICE guidance for second-line options demands different combinations of bone density and risk factors for different treatments; not only is this complex to operate in a primary care setting, but also raises difficult ethical issues, since some patients who meet the criteria for alendronate treatment but are unable to take it cannot have an alternative therapy until there is evidence of further disease progression. In contrast, NOG adopts a more pragmatic approach and does not require different criteria for second-line treatments in those who are unable to tolerate alendronate. In practice, therefore, a combination of the NICE and NOG guidance may provide the best way forward for the management of osteoporosis. An example of this approach is shown in Fig 3.

\section{Future developments and challenges}

A number of agents are currently undergoing assessment for the treatment of osteoporosis. These include new selective
Fig 3. Possible algorithm for management of osteoporosis in postmenopausal women and men aged over 50 years. The dotted arrow indicates that although further risk assessment is often indicated in postmenopausal women aged $\leqslant 75$ years and men aged over 50 years with previous fragility fracture, in some cases treatment without further assessment can be considered. $\mathrm{BMD}=$ bone mineral density; $\mathrm{CRF}=$ clinical risk factor.

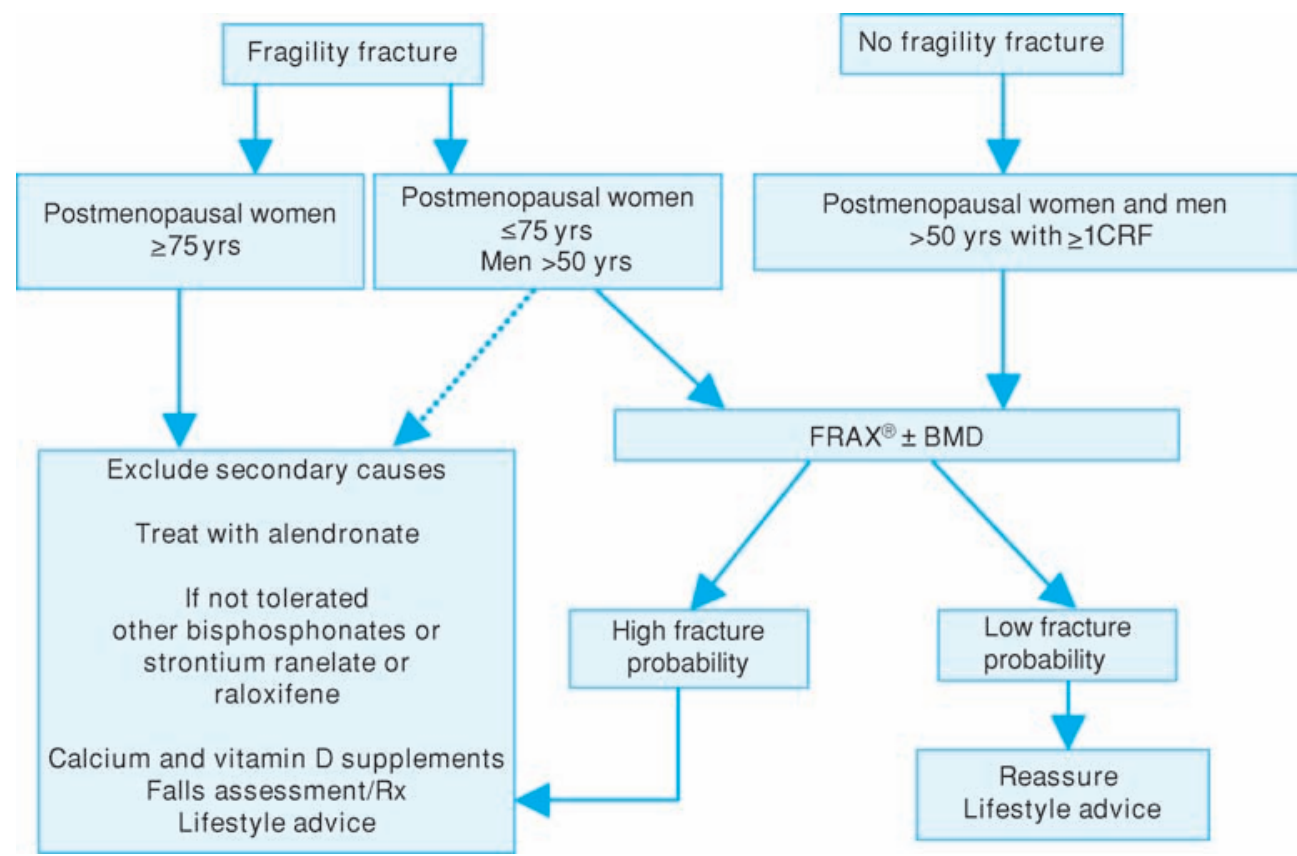


oestrogen receptor modulators, calcium sensing receptor antagonists, cathepsin $\mathrm{K}$ inhibitors and inhibitors of sclerostin, a protein produced by osteocytes that inhibits bone formation. A human monoclonal antibody to receptor activator of NFkB ligand (RANKL), a cytokine that stimulates osteoclast development and activity, has been shown in phase III trials to reduce vertebral, non-vertebral and hip fractures and is currently being evaluated by the regulatory authorities. ${ }^{18}$ It is administered once every six months by subcutaneous injection.

An important challenge for the future is to improve adherence to therapy. ${ }^{19}$ Current data indicate that $50 \%$ or fewer of patients are taking their osteoporosis medication after one year, thus reducing the potential impact of therapy on fracture burden. While the reduced dosing frequency of newer bisphosphonates may be associated with improved adherence, the causes of nonadherence and how these may be addressed needs further research. Secondly, more effective models of service delivery are required to improve the currently low rate of treatment in elderly individuals sustaining a fragility fracture. Fracture liaison services have proved effective in identifying such individuals and initiating appropriate diagnostic and therapeutic intervention, but more effective communication between secondary and primary care is required to ensure that treatment recommendations are implemented. ${ }^{20}$

\section{Acknowledgement}

JEC acknowledges the National Institute for Health Research for research support.

\section{References}

1 World Health Organization. Assessment of fracture risk and its application to screening for postmenopausal osteoporosis. Technical report series. Geneva: WHO, 1994.

2 Kanis JA. Diagnosis of osteoporosis and assessment of fracture risk. Lancet 2002;359:1929-36.

3 Kanis JA on behalf of the World Health Organization Scientific Group. Assessment of osteoporosis at the primary health-care level. Technical report. Sheffield: WHO Collaborating Centre, University of Sheffield, 2008.

4 Kanis JA, McCloskey EV, Johansson $\mathrm{H}$ et al; National Osteoporosis Guideline Group. Case finding for the management of osteoporosis with FRAX-assessment and intervention thresholds for the UK. Osteoporos Int 2008;19:1395-408.

5 Royal College of Physicians. Osteoporosis: clinical guidelines for prevention and treatment. London: RCP, 1999.

6 Royal College of Physicians and Bone and Tooth Society of Great Britain. Osteoporosis: clinical guidelines for prevention and treatment.
Update on pharmacological interventions and an algorithm for management. London: RCP, 2000.

7 Poole KE, Compston JE. Osteoporosis and its management. BMJ 2006;333:1251-6.

8 Kanis JA, Adams J, Borgstrom $\mathrm{F}$ et al. The cost-effectiveness of alendronate in the management of osteoporosis. Bone 2008;42:4-15.

9 Black DM, Delmas PD, Eastell R et al. Once-yearly zoledronic acid for treatment of postmenopausal osteoporosis. $N$ Engl J Med 2007;356:1809-22.

10 Bone HG, Hosking D, Devogelaer J-P et al. Ten years' experience with alendronate for osteoporosis in postmenopausal women. New Engl J Med 2004;350:1189-99.

11 Odvina CV, Zerwekh JE, Rao DS et al. Severely suppressed bone turnover: a potential complication of alendronate. J Clin Endocrinol Metab 2005;90:1294-301.

12 Khan AA, Sándor GK, Dore E et al. Canadian Association of Oral and Maxillofacial Surgeons. Canadian consensus practice guidelines for bisphosphonate associated osteonecrosis of the jaw. J Rheumatol 2008;35:1391-7.

13 Blake GM, Fogelman I. Theoretical model for the interpretation of BMD scans in patients stopping strontium ranelate treatment. J Bone Miner Res 2006;21:1417-24.

14 Compston JE. Skeletal actions of intermittent parathyroid hormone: effects on bone remodelling and structure. Bone 2007;40:1447-52.

15 National Institute for Health and Clinical Excellence. Final appraisal determination. Alendronate, etidronate, risedronate, raloxifene, strontium ranelate and teriparatide for the secondary prevention of osteoporotic fragility fractures in postmenopausal women. London: NICE, 2008.

16 National Institute for Health and Clinical Excellence. Final appraisal determination. Alendronate, etidronate, risedronate, raloxifene and strontium ranelate for the primary prevention of osteoporotic fragility fractures in postmenopausal women. London: NICE, 2008.

17 Compston J, Cooper A, Cooper C et al on behalf of the National Osteoporosis Guideline Group (NOGG). Guidelines for the diagnosis and management of osteoporosis in postmenopausal women and men from the age of 50 years in the UK. Maturitas 2009;62:105-8.

18 Lewiecki EM, Miller PD, McClung MR et al. AMG 162 Bone Loss Study Group. Two-year treatment with denosumab (AMG 162) in a randomized phase 2 study of postmenopausal women with low BMD. J Bone Miner Res 2007;22:1832-41.

19 Compston J, Seeman E. Compliance with osteoporosis therapy is the weakest link. Lancet 2006;368:973-4.

20 British Orthopaedic Association. Care of the patient with fragility fracture. London: BOA, 2007.

Address for correspondence: Professor J Compston, Box 157, Department of Medicine, Addenbrookes Hospital, Hills Road, Cambridge CB2 OQQ.

Email: jec1001@cam.ac.uk 